\title{
Botulinum Toxin A in the Treatment of Spinal Cord Injury Patients with Refractory Neurogenic Detrusor Overactivity
}

\author{
Ronaldo A. Alvares, Jose A. F. Silva, Andre L. Barboza, Raphael T. M. Monteiro \\ SARAH Network of Rehabilitation Hospitals, Belo Horizonte Unit, Belo Horizonte, MG, Brazil
}

\begin{abstract}
Purpose: To evaluate the efficacy of botulinum toxin type A injections in the detrusor muscle in patients with spinal cord injury and urinary incontinence due to detrusor overactivity and refractory to anticholinergic agents.

Materials and Methods: We prospectively evaluated 22 patients with spinal cord injuries, whose bladders were emptied by intermittent catheterization. All patients had detrusor overactivity and urinary incontinence that proved difficult to treat, despite using high doses of two different anticholinergics. The pre-treatment assessment included a complete urodynamic study and ultrasonography of the kidneys and urinary tract. A one-month follow-up was completed with urodynamic evaluation and the clinical response was evaluated through outpatient consultations and telephone contact.

Results: After the procedure, the maximum cystometric capacity and the bladder reflex volume increased, whereas the maximum detrusor pressure and compliance decreased. The mean duration of continence was $7 \pm 7$ months. In 18 patients $(81.8 \%)$, it was necessary to administer anticholinergics to achieve continence. Five patients $(22.7 \%)$ had indication of reinjection, and augmentation cystoplasty was indicated in 9 patients $(40.9 \%)$.

Conclusion: The use of botulinum toxin in the treatment of neurogenic detrusor overactivity refractory to anticholinergics is an option before more invasive treatments, such as augmentation cystoplasty, are attempted. In our study as well as in the literature, there was improvement in most urodynamic parameters. Overall, $40.9 \%$ of patients underwent augmentation cystoplasty and $81.8 \%$ of patients needed anticholinergic agents to reach urinary continence. Further studies are necessary to improve the procedure and to achieve better clinical results.
\end{abstract}

Key words: neurogenic bladder; spinal cord injury; botulinum toxin A, anticholinergic refractory

Int Braz J Urol. 2010; 36: 732-7

\section{INTRODUCTION}

Suprasacral spinal cord lesions induce neurogenic detrusor overactivity and concurrent detrusor sphincter dyssynergia that impairs the storage and emptying functions of the urinary bladder. The subsequent high intravesical pressure leads to reduced bladder capacity and incontinence and induces potential structural damage to the bladder wall and upper urinary tract. Current treatment options rely first on emptying the bladder by clean intermittent catheterization and on oral anticholinergic medication to reduce the bladder pressure and increase bladder capacity (1). In the case of a lack of efficacy or severe side effects from the oral anticholinergic agents, surgical procedures such as augmentation cystoplasty appear to be effective long term solutions for many patients (2). However, due to its invasive nature, surgery is only considered when conservative treatment has failed or is not tolerated. 
Botulinum toxin was described by Van Ermengem (3) in 1897, and it has evolved from a potent biologic poison to a versatile clinical tool with an expanding list of uses. Botulinum toxin exists as seven serotypes, designated A, B, C, D, E, F, and G (4). At present, serotypes A and B are available for clinical use. Injected into muscle, botulinum toxin causes flaccid paralysis by inhibiting the release of acetylcholine at the presynaptic cholinergic junction. The clinical effect of botulinum toxin is transient and dose related. In smooth muscles, Smith et al. (5) have demonstrated the effect of botulinum toxin type A on acetylcholine and norepinephrine release from the bladder and urethra, respectively.

The botulinum toxin type A injection into the detrusor muscle for treatment of neurogenic detrusor overactivity was introduced in 2000. This therapy is a minimally invasive treatment option positioned between oral anticholinergic treatment that was ineffective or not tolerated and invasive surgery (6). Its safety and efficacy have been confirmed in a randomized placebo-controlled study (7). Several studies have evaluated the use of botulinum toxin type A injections into the detrusor muscle of spinal cord injury patients in an attempt to reduce neurogenic detrusor overactivity, increase bladder capacity, and reduce urge incontinence $(6,8)$. In our practice, we have observed differing clinical responses following the use of this drug, despite the improvements in most urodynamic parameters.

We evaluated the effect of botulinum-A toxin injection in the detrusor overactivity in spinal cord injury patients, clinical response correlation and implications for other treatments.

\section{MATERIALS AND METHODS}

A total of 22 patients with spinal cord injuries (16 traumatic patients and 6 non-traumatic patients), whose bladders were emptied by intermittent catheterization, had detrusor overactivity and urinary incontinence that proved difficult to treat, despite using high doses of two different anticholinergics. These patients were included in our prospective study between January 2006 and January 2009.

All methods and definitions were based on the standardization of terminology of lower urinary tract function (9). The study was done in accordance with the Ethics Committee of our institution. Written informed consent was obtained from all patients.

Patient evaluations included a complete medical history, physical examination, ultrasonography of the kidneys and urinary tract and an urodynamics assessment (Multichannel urodynamics studies Medtronic Duet systems, version 8.20, Minneapolis, MN, USA) before botulinum-A toxin injection. Maximum cystometric bladder capacity corresponded to the volume at which involuntary voiding occurred, urinary loss started or filling was stopped. The urodynamic parameters measured included reflex volume, maximum detrusor pressure during voiding, bladder compliance, and maximum cystometric bladder capacity. Reflex volume is the infused volume that induces the first detrusor contraction. Bladder compliance is calculated by the change in volume divided by the change in detrusor pressure.

All procedures were done on an outpatient basis using general anesthesia. Perioperative antibiotics were administered orally for seven days, according to urine culture, and the botulinum toxin A injection performed on the fifth day of drug administration. All patients exhibited bacteriuria. Botulinum-A toxin (Alergan) was diluted in sterile normal saline to a final concentration of 10 units $/ \mathrm{mL}$. Using a Storz cystoscope $19 \mathrm{~F}$ and 23 gauge needle, a total of 300 IU $(30 \mathrm{~mL})$ was injected at 30 detrusor muscle sites sparing the trigone as described by Schurch et al. (6). Patients were instructed to progressively taper and then discontinue their anticholinergic medications within the first 3 weeks following the procedures. The response was considered effective when a patient sustained 4 months without urinary losses even while using anticholinergic agents.

A clinical and urodynamic follow-up was obtained at 4 weeks after treatment. Subsequently, the patients returned to our practice if urinary losses reoccurred. Patients who did not return until January 2009 were contacted by telephone to assess their responses to the botulinum toxin. Further injection of toxin was indicated for patients who remained continent for 4 months or more. Augmentation cystoplasty was indicated for non-responsive cases or those in which the effects lasted less than 4 months. 


\section{RESULTS}

A total of 22 patients, 19 males and 3 females aged to 13 to 61 years $(33.6 \pm 13.6)$ with refractory neurogenic incontinence participated in our study. All patients had urinary incontinence that was refractory to anticholinergic treatment (high doses of two anticholinergic drugs). According to impairment 16 were paraplegic, six tetraplegic.

All procedures were performed with general anesthesia and were well tolerated. There were no acute complications related to the injections, such as gross hematuria, injury to adjacent structures, autonomic dysreflexia, or urinary tract infection. No complications possibly related to toxin, such as dysphagia, diplopia, or general paralysis of remote musculature occurred.

The ultrasonography of the urinary tract showed 15 patients with normal results $(68.2 \%), 5$ who had hydronephrosis $(22.7 \%), 1$ with nephropathy $(4.5 \%)$ and 1 with a parapielic cyst $(4.5 \%)$. Of the 15 patients with normal exams, 9 obtained good responses, and the 5 subjects with hydronephrosis presented only 1 patient with a good response. The patient with nephropathy did not respond and the patient with a parapielic cyst showed a good response.

Thirteen patients $(59 \%)$ were completely continent, including two patients whose clinical responses lasted only two months and were considered not effective. At the four-week follow-up, four patients with good responses had discontinued anticholinergic medications. Among the continent patients, seven needed anticholinergic agents to maintain continence. However, the amount of anticholinergic medication that they needed to become continent was less than their pre-botulinum treatment dosages. Eleven patients were incontinent despite baseline anticholinergic agent therapy and were considered failures. Nine patients (40.9\%) underwent augmentation cystoplasty.

At the 4-week follow-up, urodynamic evaluation revealed increases in mean reflex volumes from $175 \pm 79 \mathrm{~mL}$ to $312 \pm 227 \mathrm{~mL}(\mathrm{P}=0.010)$ and mean maximum cystometric bladder capacity from $219 \pm$ $113 \mathrm{~mL}$ to $404 \pm 216 \mathrm{~mL}(\mathrm{p}=0.001)$. The compliance decreased from $30.6 \pm 23.8 \mathrm{~mL} / \mathrm{cm} \mathrm{H} 2 \mathrm{O}$ to $22.2 \pm$ $11.9 \mathrm{~mL} / \mathrm{cm} \mathrm{H} 2 \mathrm{O}(\mathrm{p}=0.067)$. Detrusor overactivity decreased from $78 \pm 27 \mathrm{~cm} \mathrm{H} 2 \mathrm{O}$ to $49 \pm 30 \mathrm{~cm}$ $\mathrm{H} 2 \mathrm{O}$ (Table-1). Nine patients remained incontinent at one month following treatment, despite the fact that urinary losses had decreased, and these patients experienced improvements in all urodynamic parameters. Nine patients remained continent for more than six months. Therefore, we believe that the treatment was not effective in 11 patients (nine with incontinence and two with only short-lived responses).

\section{COMMENTS}

Botox injections into the detrusor provide clinically significant improvement in patients with neurogenic detrusor overactivity refractory to antimuscarinics and are very well tolerated (7). In our study, we observed continence during a period of more than 4 months in $50 \%$ of patients undergoing treatment, while $81.8 \%$ of patients continued anticholinergic therapies to achieve continence. In other studies with similar populations of patients, the percentage of continents after toxin injection ranged from $42-87 \%$

Table 1 - Urodynamic parameters before and after botulinum toxin A injection.

\begin{tabular}{|c|c|c|c|c|}
\hline Urodynamic Parameters & & Before & After & p Value * \\
\hline Maximum cystometric bladder capacity (mL) & Mean (SD) & $219(113)$ & $404(216)$ & 0.001 \\
\hline Detrusor overactivity $(\mathrm{cm} / \mathrm{H} 2 \mathrm{O})$ & Mean (SD) & $78(27)$ & $49(30)$ & 0.001 \\
\hline Reflex volume (mL) & Mean (SD) & $175(79)$ & $312(227)$ & 0.010 \\
\hline Compliance & Mean (SD) & $31(24)$ & $22(12)$ & 0.067 \\
\hline
\end{tabular}

* Standard deviation. 
and $28-58 \%$ discontinued use of anticholinergics (10).

In our study, we observed decreased of bladder compliance in urodynamic parameters after botulinum toxin injection, in contrast to other reports in the literature (10). In our opinion, this could occur because some cases have low rates of compliance associated with overactivity not previously identified. This may bias results, as treating the neurogenic detrusor overactivity can not only lead to gains in bladder capacity function but also to a loss of compliance that may be more evident in the urodynamic study.

There are some reported limitations with respect to botulinum A toxin injections into the detrusor. Most studies, as well as our own, were small-scale studies enrolling fewer than 50 patients. The doses of anticholinergics considered high in patients who are refractory to medical treatment are not well defined. We believe that some cases could also benefit from higher doses of these drugs. In our study, we included only cases that used at least two anticholinergic agents in high doses with no satisfactory outcomes. Those cases may have a more difficult clinical solution.

Although there is no consensus regarding the optimal dose of botulinum toxin or a standard injection procedure in the detrusor muscle, most studies have used the technique described by Schurch et al. at a dose of $300 \mathrm{IU}(6,11)$. Some studies such as Kuo et al. have showed similar responses at doses of 200 IU. However, this study was conducted in patients with neurogenic detrusor overactivity and idiopathic detrusor overactivity (12). Recently Gomes et al. suggested that different pharmaceutical formulations of botulinum toxin A might influence the final results. Although further evaluation will be required to assess these formulations (13). Regarding the injection technique, it is important to consider that the neurogenic bladder wall has varying levels of thickness. This may lead to inadvertent injection of the medication outside the bladder wall, even in procedures performed by the same surgeon using standard endoscopy materials. Additionally, the injection can often occur in a subendothelial region. The Kuo study showed that there was no difference in response in this instance, but the patient cohort was of limited size (14). In our study, as well as oth- ers, we recommended botulinum toxin injection in accordance with the clinical response to treatment (incontinence despite anticholinergic in high doses) and urodynamic results. Our results were not as satisfactory as other studies, probably because of the fact that many of these patients presented bladders with major structured deformities such as a large number of diverticula, low capacity and significant changes in their shape. These bladder morphology variations have been observed during the procedure as well as in cystography. Currently, we are studying the role of bladder morphology in the light of the results of botulinum toxin A injection into detrusor.

Despite the small number of patients in our study, those with hydronephrosis experienced less satisfactory responses, a finding that may help in identification of patients to be treated. We have also compared the urodynamic parameters before and after botulinum toxin A injection separately in patients who had good response and those in which the response was not satisfactory (Table-2). We observed that urodynamic parameters were similar before the procedure, although the response was different in the two groups. This allows us to conclude that urodynamic evaluation does not predict how will be the response to the procedure.

We observed that $50 \%$ of patients had no satisfactory responses to treatment as defined by sustained continence for more than 4 months. Although we observed an improvement in most urodynamic parameters of these patients, these improvements were not sufficient to obtain an adequate bladder capacity to remain continent, or did not have a satisfactory duration. This explains why nine patients underwent augmentation cystoplasty. We need to define a more precise parameter for identifying patients with neurogenic bladder dysfunction who would benefit from the use of botulinum toxin, thereby avoiding treatment in some patients who would be more appropriately treated with the augmentation cystoplasty. Another factor we must consider is that the use of botulinum toxin on neurogenic bladder dysfunction refractory to medical treatment is relatively recent. As this kind of treatment has many unknowns, this may present some difficulties in the light of patient acceptance, as it is not a definitive treatment and requires regular injections. 
Table 2 - Urodynamic parameters (mean \pm standard deviation) in patients with response not satisfactory and with good response before and after botulinum toxin A injection.

\begin{tabular}{lcccc}
\hline & \multicolumn{2}{c}{ Response Not Satisfactory } & \multicolumn{2}{c}{ Good Response } \\
& Before & After & Before & After \\
\hline Maximum cystometric bladder capacity $(\mathrm{mL})$ & $230(105)$ & $322(177)$ & $202(128)$ & $521(222)$ \\
Detrusor overactivity $(\mathrm{cm} / \mathrm{H} 2 \mathrm{O})$ & $69(22)$ & $60(34)$ & $92(28)$ & $32(8)$ \\
Reflex volume $(\mathrm{mL})$ & $190(77)$ & $186(79)$ & $153(82)$ & $494(251)$ \\
Compliance & $31.7(26.8)$ & $19.5(10.5)$ & $29.0(19.9)$ & $26.1(13.2)$ \\
\hline
\end{tabular}

Also, note that most patients require anticholinergics to achieve and maintain continence. We believe that the effect of this therapy should last a least 4 months and that the procedure should be performed up to 3 times a year. We believe that a greater number of procedures per year would be impracticable, especially for patients who require treatment for an indefinite period, but we also agree that it is not a factor that contraindicates a new injection of medication.

\section{CONCLUSION}

The use of botulinum toxin in the treatment of neurogenic detrusor overactivity refractory to anticholinergics is an option to consider before more invasive treatments, such as augmentation cystoplasty, are implemented. In our study as well as in the literature, there was improvement in most urodynamic parameters analyzed following botulinum toxin injection. Overall, $40.9 \%$ of patients underwent augmentation cystoplasty and $81.8 \%$ of patients continued to take anticholinergic agents to reach urinary continence. Further studies are necessary to improve the procedure and to achieve better clinical results.

\section{CONFLICT OF INTEREST}

None declared.

\section{REFERENCES}

1. Abrams P, Larsson G, Chapple C, Wein AJ: Factors involved in the success of antimuscarinic treatment. BJU Int. 1999; 83 (Suppl 2):42-7.

2. Chartier-Kastler EJ, Mongiat-Artus P, Bitker MO, Chancellor MB, Richard F, Denys P: Long-term results of augmentation cystoplasty in spinal cord injury patients. Spinal Cord. 2000; 38: 490-4.

3. van Ermengem E: Classics in infectious diseases. A new anaerobic bacillus and its relation to botulism. E. van Ermengem. Originally published as "Ueber einen neuen anaëroben Bacillus und seine Beziehungen zum Botulismus" in Zeitschrift für Hygiene und Infektionskrankheiten 26: 1-56, 1897. Rev Infect Dis. 1979; 1: 701-19.

4. Comella CL, Pullman SL: Botulinum toxins in neurological disease. Muscle Nerve. 2004; 29: 628-44.

5. Smith CP, Franks ME, McNeil BK, Ghosh R, de Groat WC, Chancellor MB, et al.: Effect of botulinum toxin A on the autonomic nervous system of the rat lower urinary tract. J Urol. 2003; 169: 1896-900.

6. Schurch B, Stöhrer M, Kramer G, Schmid DM, Gaul G, Hauri D: Botulinum-A toxin for treating detrusor hyperreflexia in spinal cord injured patients: a new alternative to anticholinergic drugs? Preliminary results. J Urol. 2000; 164: 692-7.

7. Schurch B, de Sèze M, Denys P, Chartier-Kastler E, Haab F, Everaert K, et al.: Botulinum toxin type a is a safe and effective treatment for neurogenic urinary incontinence: results of a single treatment, randomized, placebo controlled 6-month study. J Urol. 2005; 174: 196-200. 
8. Schurch B, Denys P, Kozma CM, Reese PR, Slaton T, Barron RL: Botulinum toxin A improves the quality of life of patients with neurogenic urinary incontinence. Eur Urol. 2007; 52: 850-8.

9. Abrams P, Cardozo L, Fall M, Griffiths D, Rosier P, Ulmsten U, et al.: The standardisation of terminology in lower urinary tract function: report from the standardisation sub-committee of the International Continence Society. Urology. 2003; 61: 37-49.

10. Karsenty G, Denys P, Amarenco G, De Seze M, Gamé $X$, Haab F, et al.: Botulinum toxin A (Botox) intradetrusor injections in adults with neurogenic detrusor overactivity/neurogenic overactive bladder: a systematic literature review. Eur Urol. 2008; 53: 275-87.

11. Rapp DE, Lucioni A, Bales GT: Botulinum toxin injection: a review of injection principles and protocols. Int Braz J Urol. 2007; 33: 132-41.
12. Kuo HC: Urodynamic evidence of effectiveness of botulinum A toxin injection in treatment of detrusor overactivity refractory to anticholinergic agents. Urology. 2004; 63: 868-72.

13. Gomes CM, de Castro Filho JE, Rejowski RF, TrigoRocha FE, Bruschini H, de Barros Filho TE, et al.: Experience with different botulinum toxins for the treatment of refractory neurogenic detrusor overactivity. Int Braz J Urol. 2010; 36: 66-74.

14. Kuo HC: Will suburothelial injection of small dose of botulinum A toxin have similar therapeutic effects and less adverse events for refractory detrusor overactivity? Urology. 2006; 68: 993-7; discussion 997-8.

\section{Correspondence address:}

Dr. Ronaldo Alvarenga Álvares

Rede Sarah de Hospitais de Reabilitação

Av. Amazonas 5953, Gameleira

Belo Horizonte, MG, 30510-000,Brazil

E-mail: ronaldoalvares@sarah.br 